\title{
COMPROMISO MUSCULAR POR LINFOMA NO HODGKIN: CASO CLÍNICO Y REVISIÓN DEL TEMA
}

\author{
Drs. Pablo Avaria $P^{(1)}$, Claudia Ortega $M^{(2)}$, Rossana Pruzzo $C^{(3)}$, Alejandra Pizarro $G^{(2)}$, Horacio Amaral $P^{(4)}$.
}

1. Médico Cirujano, Servicio Urgencia Hospital Militar de Santiago de Chile.

2. Médico Radiólogo. Servicio Medicina Nuclear PET/CT, Fundación Arturo López Pérez, Santiago - Chile.

3. Médico Nuclear. Servicio Medicina Nuclear PET/CT, Fundación Arturo López Pérez, Santiago - Chile.

4. Médico Nuclear, Jefe de Servicio Medicina Nuclear PET/CT, Fundación Arturo López Pérez, Santiago - Chile.

\section{MUSCLE INFILTRATION BY NON-HODGKIN LYMPHOMA: A CASE AND BIBLIOGRAPHIC REVIEW}

Abstract: A case report of a patient with treated Non-Hodgkin Lymphoma is presented. In his usual tomographic control patient was requested a PET-CT scan to supplement prior study that showed a metabolically active focus on the left adductor muscle without evident tomographic correlation. Lesion underwent both a soft tissue ultrasound study and a directed biopsy, the latter being positive for secondary infiltration by lymphoma. This case has demonstrated the usefulness of applying complementary techniques in the management of these lesions, mainly of PET-CT scans in the study of unusual sites of spread. This combined medical imaging technique allows accurate lesion localization, which in turn permits performance of a subsequent directed study.

Keywords: PET-CT imaging, Non-Hodgkin lymphoma, Muscle.

Resumen: Se presenta un caso clínico de paciente con linfoma no Hodgkin tratado; en control tomográfico habitual se le solicitó PET-CT para complementar su estudio, que demuestra un foco metabólicamente activo en el espesor de músculo aductor izquierdo sin traducción tomográfica evidente. La lesión fue estudiada con ultrasonografia de partes blandas y biopsia dirigida, que resultó positiva para infiltración secundaria por linfoma. Este caso demuestra la utilidad de las técnicas complementarias en el manejo de estos pacientes, en especial la utilidad del PET-CT en el estudio de sitios inhabituales de diseminación. En este examen existe la posibilidad de identificar con precisión la localización de las lesiones mediante la

Avaria $\boldsymbol{P}$ y cols. Compromiso muscular por linfoma no Hodgkin: Caso clínico y revisión del tema. Rev Chil de Radiol 2010; 16(2): 53-58.

Correspondencia: Dra. Claudia Ortega $M$. cortegamogi@gmail.com • pmelocastillo@gmail.com Trabajo recibido el 27 de julio de 2009, aceptado para publicación el 15 de marzo de 2010.
TC complementaria, lo que permite efectuar posteriormente el estudio dirigido.

Palabras clave: PET-CT, linfoma no Hodgkin, músculo.

\section{Introducción}

El linfoma es la neoplasia primaria del sistema linforreticular; ha sido clasificada en linfoma de Hodgkin (LH) y linfoma no Hodgkin (LNH), que corresponden a entidades totalmente distintas desde el punto de vista clínico, histológico y en su respuesta a la terapia. En ambos casos, el grado de compromiso nodal y/o extranodal determinará el estadío clínico de la enfermedad, definido por la clasificación de Ann Arbor (Tabla I) ${ }^{(1-7)}$.

Tabla I. Clasificación clínica.

Linfoma de Hodgkin y No Hodgkin (Ann Arbor)

Etapa* Criterio

I Una sola región ganglionar o sitio extralinfático (IE).

II Dos o más regiones ganglionares al mismo lado del diafragma o un órgano extralinfático y sus ganglios linfáticos regionales al mismo lado del diafragma (IIE).

III Ganglios a ambos lados del diafragma, incluido el bazo.

IV Compromiso de uno o más sitios extralinfáticos (ej. médula ósea, pulmón, hígado) y compromiso ganglionar distante (no regional).

*Subclasificación E indica compromiso extranodal adyacente a un ganglio (ej, enfermedad de ganglios mediastínicos e hiliares con infiltración pulmonar adyacente, se clasifica como etapa IIE). La etapa puede ser A, para indicar la ausencia o $\mathrm{B}$ la presencia de síntomas constitucionales (baja peso, fiebre o sudoración nocturna). Estos ocurren generalmente en etapas III y IV (20-30\%); el sufijo X se usa en enfermedad voluminosa o "bulky", > $10 \mathrm{~cm}$ diámetro máximo $0>1 / 3$ de diámetro torácico en $R x$ tórax. 
El diagnóstico de linfoma se realiza en base a estudio histológico; los exámenes imaginológicos son básicos para la etapificación, re-etapificación y control de terapia, entre los cuales la tomografía computada (TC) y actualmente la tomografía por emisión de positrones asociada a tomografía computada (PET-CT) son las técnicas de elección ${ }^{(8)}$.

El linfoma puede comprometer cualquier parte del sistema músculo esquelético. Los linfomas músculo esqueléticos primarios son raros, siendo más comunes las manifestaciones por infiltración secundaria; en ambos casos la presentación radiológica es variada ${ }^{(9-11)}$.

\section{Caso clínico}

Paciente de 50 años, sexo masculino, portador de linfoma no Hodgkin indolente de células $B$ CD$20(+)$, diagnosticado y tratado con R-CHOP en el año 2002. Antecedente de recaída cervical en el año 2006, tratada con quimioterapia (Rituximab) hasta agosto 2007. Se solicita PET-CT para re-etapificación post-terapia.

El examen se realiza 50 minutos después de la inyección endovenosa de $9 \mathrm{mCi}$ de 2-(18F)-fluoro2-desoxi-D-glucosa(FDG), obteniéndose imágenes de PET-CT simultáneas desde la base del cráneo a los muslos. Estas fueron fusionadas y corregidas por atenuación. En el momento previo a la inyección del trazador la glicemia era normal. Se realiza TC de cuello, tórax, abdomen y pelvis, posterior a la administración de medio de contraste yodado endovenoso.

El examen demuestra un foco intensamente hipermetabólico en relación al músculo aductor izquierdo (SUV max. 15,7) que no tiene una clara correlación tomográfica (Figuras 1-3).

El resto de las imágenes analizadas muestran captación fisiológica de 18FDG en cerebro, glándulas salivales, cuerdas vocales, estructuras musculares, miocardio, hígado, bazo, tracto intestinal y sistema urogenital.

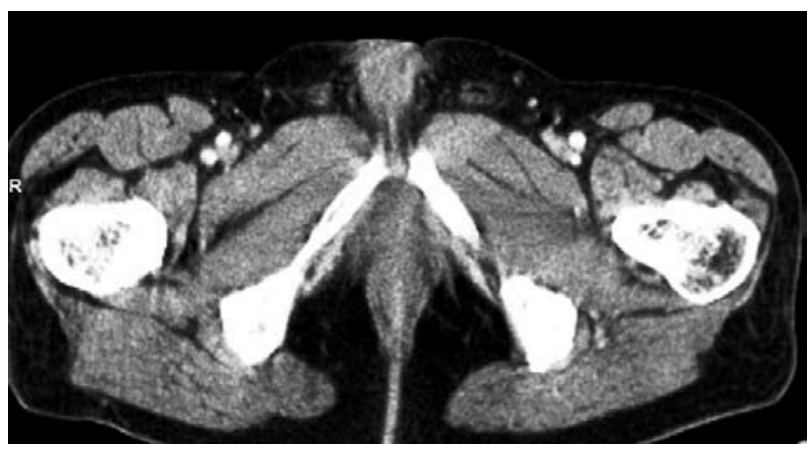

Figura 1. Imagen axial de tomografía computada multicorte en región pubiana, con uso de contraste endovenoso que demuestra una leve mayor captación de contraste en relación al músculo aductor izquierdo.

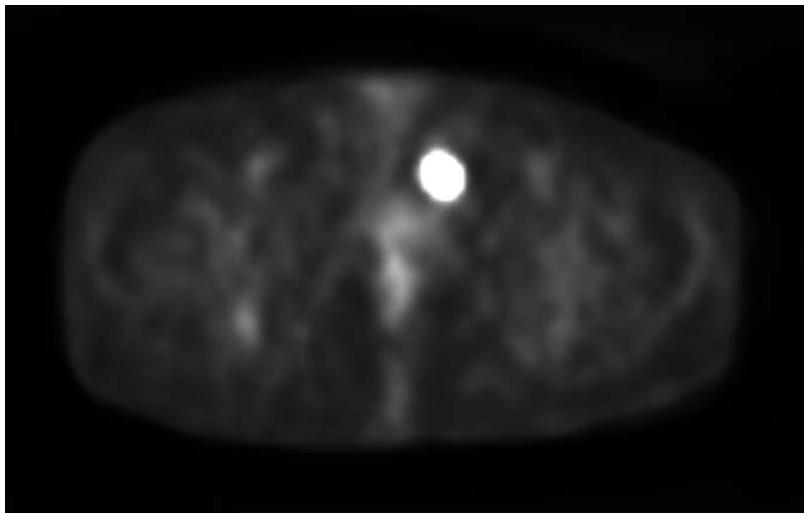

Figura 2. Corte axial de imagen PET, demuestra foco hipercaptante, que anatómicamente se ubica a nivel de musculatura aductora izquierda.
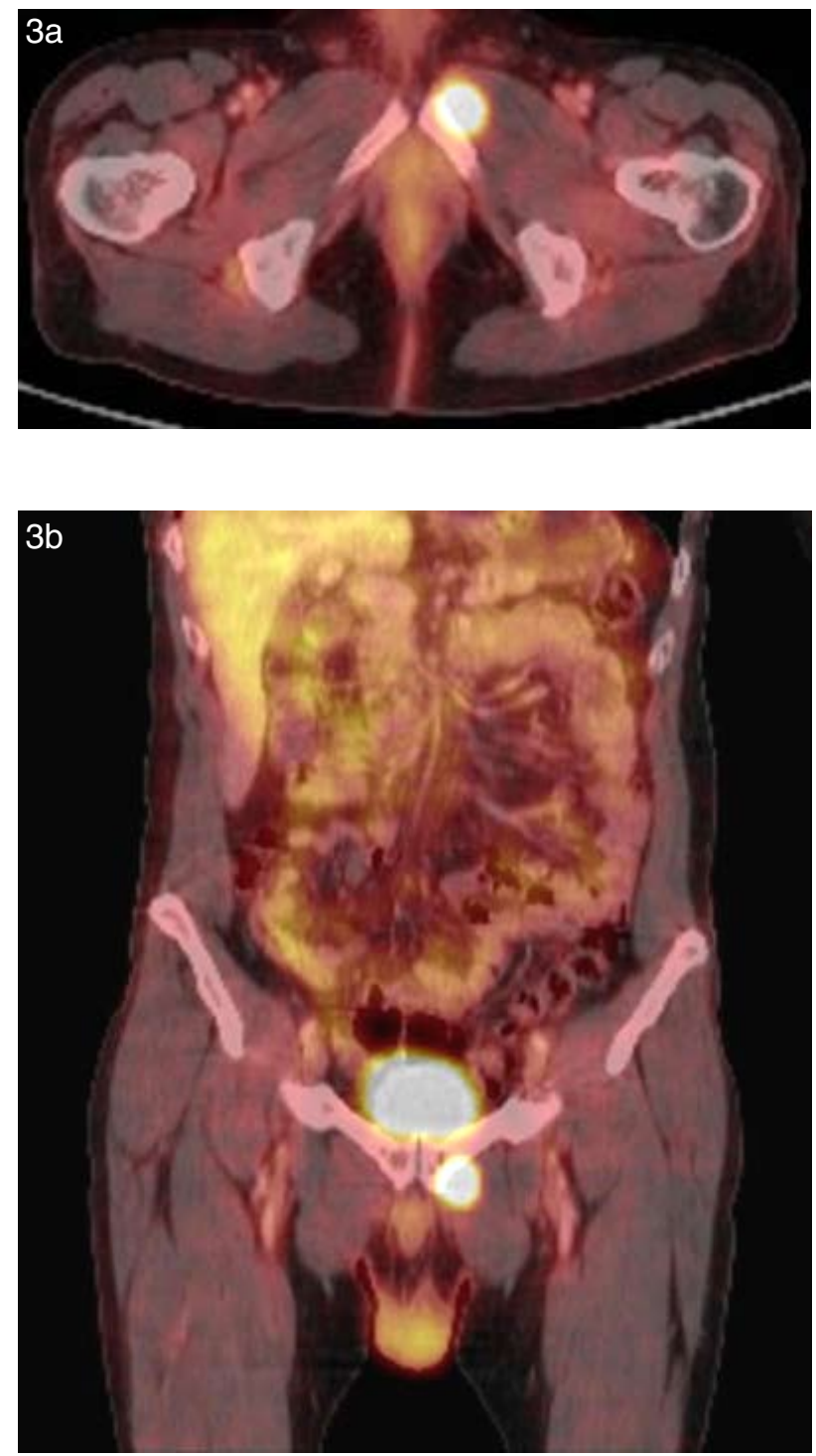

Figura 3. Imágenes fusionadas de PET-CT en reconstrucciones axial (a) y coronal (b), que demuestran la presencia de un intenso foco hipermetabólico en relación al músculo aductor izquierdo (SUV max. 15.7). 
Se complementó el estudio con ultrasonografia (US) de partes blandas en otro centro (Figura 4), que muestra un nódulo bien definido, hipoecogénico, de $10 \times 17 \mathrm{~mm}$ en el espesor del músculo aductor izquierdo, en correlación con el foco visualizado en PET-CT.

Se realizó biopsia guiada por US, obteniéndose trozos de tejido muscular de aproximadamente $2.5 \mathrm{cms}$. La biopsia confirmó infiltración del tejido blando por tumor maligno escasamente diferenciado, con inmunohistoquimica positiva para antígeno leucocitario común y CD20, que arrojan intenso positivismo en las membranas citoplasmáticas, por lo que es concluyente de infiltración por linfoma no Hodgkin variedad $\mathrm{B}$, similar al diagnosticado previamente en este paciente.

\section{Linfoma no Hodgkin}

Los pacientes con LNH suelen debutar con enfermedad más avanzada que los pacientes con LH. La diseminación puede no ser por contigüidad y afectar localizaciones extra ganglionares. La enfermedad intratorácica aparece en aproximadamente el 40 a $50 \%$ de los pacientes. En general, las cadenas ganglionares del mediastino superior no están afectadas, siendo más frecuente la afección pulmonar, pleural, mediastínica posterior, de ángulos cardiofrénicos e hiliar.

Aunque los linfomas son neoplasias predominantemente de nodos linfáticos, puede ocurrir compromiso extranodal, principalmente en aquellos con arquitectura difusa, ya sea como manifestación primaria de la enfermedad o por diseminación secundaria, siendo ésta la más frecuente ${ }^{(12-14)}$.

El compromiso extranodal en el LNH está bien documentado, siendo los sitios de compromiso más frecuente el hígado, anillo de Waldeyer y el tracto gastrointestinal (GI)(15).

\section{Estudio imaginológico en linfoma}

Los estudios se dividen básicamente en tres categorías:

1) Estudios anatómicos: radiografía de tórax, ultrasonografía, TC y resonancia magnética. Dentro de éstos, la TC es la técnica de elección por su mayor costo-efectividad en relación a las otras modalidades.

2) Estudios funcionales: Cintigrafía con galio 67 y tomografía por emisión de positrones (PET) con glucosa marcada (18F-FDG), donde el objetivo es evaluar el metabolismo 0 actividad tumoral.

3) Combinación de ambos (PET-CT), donde la fusión de imágenes obtenidas simultáneamente permite localizar las lesiones con una mayor certeza diagnóstica ${ }^{(8,10,11)}$.
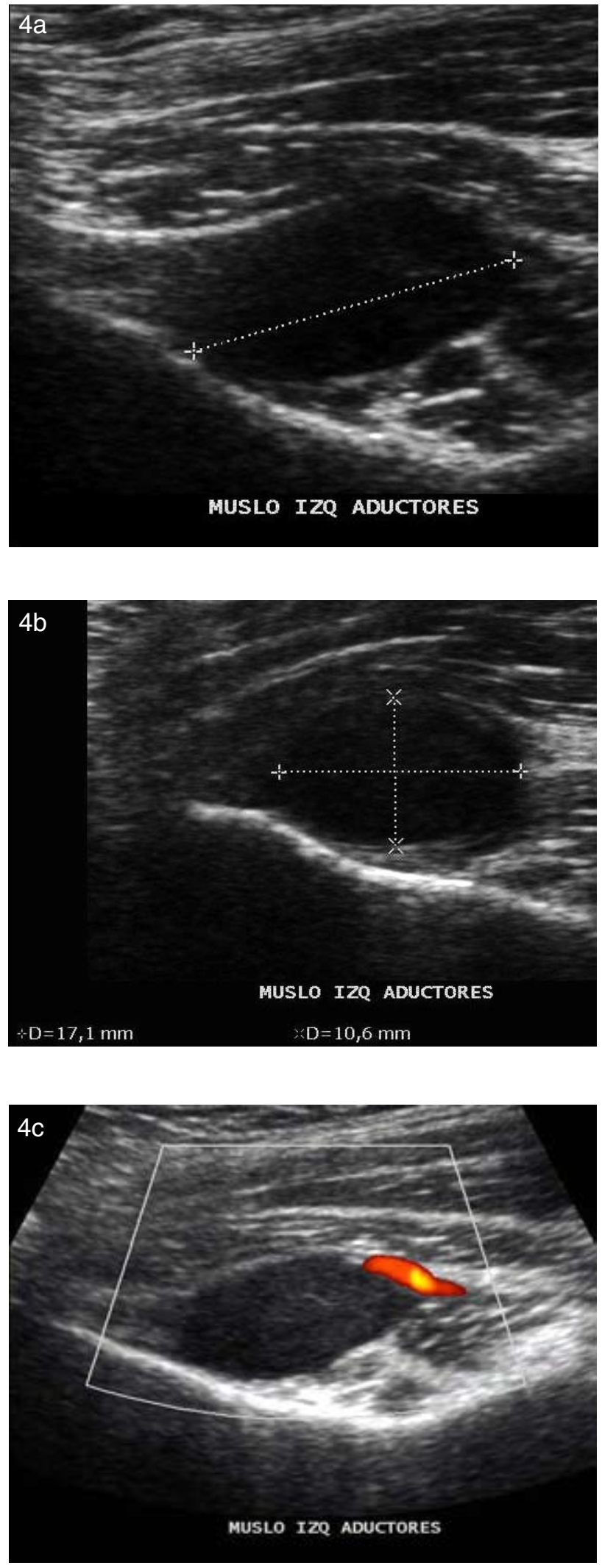

Figura 4. Ultrasonografía de partes blandas de la región pubiana izquierda demuestra imagen nodular hipoecogénica, homogénea de $17 \times 10 \mathrm{~mm}$ en el espesor del músculo aductor izquierdo. a) Corte longitudinal, b) transversal, c) con Doppler color se evidencia escaso flujo vascular en la periferia de la lesión. 


\section{Tomografía Computada (TC)}

La TC es el estudio de elección para la evaluación de los linfomas de Hodgkin y no-Hodgkin. Su rol se puede resumir como sigue ${ }^{(1,10,12,16)}$ :

a. Definir la extensión completa para una adecuada etapificación.

b. Asistir en la planificación del tratamiento

c. Evaluar la respuesta a tratamiento

d. Monitorizar el progreso del paciente y posibles recaídas.

\section{Ultrasonido (US)}

Se utiliza habitualmente en el estudio de linfoadenopatías de cadenas ganglionares a nivel axilar, inguinal o cervical. EI US de partes blandas permite evaluar compromiso muscular con buen rendimiento, debido a la diferencia en la ecogenicidad de los tumores en comparación con los tejidos normales circundantes ${ }^{(17)}$.

Por otro lado, tiene la ventaja de poder evaluar la masa tumoral en sentido transverso y longitudinal, dando una impresión más correcta de la configuración de la lesión y el volumen de ésta. Además, el US es capaz de detectar tumores pequeños que pueden no ser visualizados mediante otros métodos, como por ejemplo la TC.

\section{PET y PET-CT}

EI PET 18F-FDG es una técnica de estudio metabólico, que se basa en la capacidad de este trazador de incorporarse a las células normales y tumorales de la misma forma en que lo hace la molécula de glucosa.

Desde su introducción en la práctica clínica, el PET con 18F-FDG ha demostrado ser altamente sensible para la detección de focos hipermetabólicos potencialmente neoplásicos, ya que en estos tejidos el consumo de glucosa se encuentra marcadamente aumentado ${ }^{(18-30)}$.

La técnica del PET-CT permite la adquisición simultánea de estudios metabólico y anatómico, fusionando posteriormente estas imágenes. La integración de la alta sensibilidad del PET para identificar lesiones neoplásicas hipermetabólicas con la adecuada resolución anatómica de la TC, permite localizar con gran precisión los focos hipercaptantes de 18F-FDG en las estructuras anatómicas, lo que entrega mayor y mejor información que la obtenida con ambos estudios por separado.

Aún existen pocos estudios que demuestran el real impacto del uso el PET-CT en la etapificación y re-etapificación de los linfomas. Sin embargo, algunas series muestran sensibilidad para detectar compromiso nodal de $94 \%$ para el PET-CT y $88 \%$ para la TC (con uso de medio de contaste ev). La especificidad para ambas técnicas es de $100 \%$ y $86 \%$ respectivamente. Para determinar compromiso extranodal, se registran sensibilidades de $88 \%$ para el PET-CT y $50 \%$, para TC, con especificidad de 100 y $90 \%$, respectivamente. La certeza diagnóstica del PET-CT es de $93 \%$ versus $84 \%$ para $P E T$, en linfoma ${ }^{(25)}$.

EI PET con 18F-FDG es más sensible que el estudio con Ga67 citrato, detectando mayor número de lesiones y pacientes con alteraciones. El galio 67 tiene además la desventaja de que no se produce en Chile por lo que debe importarse, demorando entre 7 y 15 días. Dada su excreción intestinal, se puede subestimar o sobreestimar el compromiso abdominal de linfoma ${ }^{(35)}$.

La asociación de la imagen metabólica a la morfológica aumentó la certeza diagnostica del PETCT con FDG ${ }^{(36-41)}$.

\section{Indicaciones de PET-CT en linfoma}

Según el consenso del grupo internacional del linfoma, el PET-CT, se recomienda (no obligatorio), en las siguientes situaciones ${ }^{(26)}$ :

1. Etapificación:

- Linfoma Hodgkin: Útil para diferenciar estadíos tempranos (I y II) de etapas avanzadas (III y IV) de la enfermedad.

- Linfoma no Hodgkin: La utilidad está determinada por el tipo histológico del linfoma, siendo altamente sensible en el LNH difuso de células grandes B (DLBCL), linfoma folicular y linfoma del manto.

2. Control de tratamiento:

Numerosos estudios han demostrado la utilidad de realizar un control con PET-CT interciclos, al término del primer o cuarto ciclo de quimioterapia con multiagentes para predecir la respuesta a terapia y controlar evolución de la enfermedad. Sin embargo, no existen aún datos estadísticamente significativos para demostrar mejoría en los resultados que permitan alterar el tratamiento basándose en estos resultados. La práctica de PET-CT interciclos será restringida a proyectos de investigación clínicos hasta obtener resultados estadísticamente significativos.

\section{Evaluación de masa residual:}

Una de las principales indicaciones del PET-CT es la evaluación de respuesta al finalizar la terapia, especialmente en aquellos pacientes con masa residual mayor de $2 \mathrm{~cm}$ en mediastino o abdomen, dada la capacidad de distinguir viabilidad tumoral de necrosis o fibrosis.

EI PET-CT de control debe ser realizado al menos 3 semanas después de finalizada la quimioterapia u 8-12 semanas después de finalizada la radioterapia.

\section{Compromiso músculo esquelético}

El linfoma muscular primario (LMP) es muy raro, 
en general no supera $0,3 \%$ de los casos de LH y $1,5 \%$ de LNH. El LMP ha sido asociado a mal pronóstico y generalmente corresponde a LNH difuso de células B. En general, el compromiso muscular por linfoma ocurre secundario a diseminación hematógena de compromiso nodal.

El diagnóstico definitivo de linfoma es difícil si se utilizan solamente criterios imaginológicos, requiriendo habitualmente confirmación histológica ${ }^{(12,13,42,43)}$.

\section{Compromiso muscular}

LNH puede comprometer el músculo, ya sea como una masa definida o como infiltración difusa ${ }^{(13)}$. Si bien el compromiso puede ser primario, la diseminación secundaria de una masa adyacente es lo más frecuente.

El hallazgo imaginológico habitual es la infiltración tumoral difusa. El compromiso por linfoma generalmente se presenta en US como una lesión hipoecogénica homogénea, con flujo vascular al estudio Doppler color. Puede manifestarse como grandes masas, nódulos confluentes, pequeños nódulos diseminados o también como miositis y paniculitis. Estos hallazgos requieren biopsia dirigida en la mayoría de los casos ${ }^{(33)}$. La TC presenta bajo rendimiento en la evaluación del compromiso muscular por linfoma, solamente con la administración de contraste es posible distinguir tenues diferencias de densidad que lo sugieran.

En RM, las lesiones aparecen de señal intermedia en T1 y un alto porcentaje evidencian señal intermedia en T2.

En la mayoría de los casos la lesión se ubica justo por debajo de la fascia con extensión subcutánea; la mitad de los casos presenta compromiso de más de un compartimiento y en ocasiones se observa extensión del tumor a través de trayectos neurovasculares ${ }^{(34)}$. En los casos de infiltración tumoral de mayor tamaño, se puede producir aumento de volumen muscular sin que exista obstrucción linfática o una masa tumoral. La mayoría de estos pacientes presentan compromiso óseo adyacente al músculo afectado. Después del tratamiento, en este grupo el tamaño muscular regresa rápidamente a la normalidad( ${ }^{(27-32)}$.

\section{Conclusiones}

Múltiples publicaciones confirman la certeza diagnostica del PET y PET/CT en la etapificación, control de terapia y detección de recidiva de linfomas.

El LNH difuso de células grandes B presenta la mayor avidez por la glucosa y en este caso la reetapificación con PET/CT permitió seleccionar el sitio menos invasivo para biopsia.

El uso simultáneo de PET y TC genera mayor certeza diagnóstica en linfoma, comparado con ambas técnicas por separado, lo que queda ejemplificado en este caso clínico.

\section{Bibliografía}

1. Fishman EK, Kuhlman JE, Jones RJ. CT of lymphoma: spectrum of disease. RadioGraphics 1991; 11: 647669

2. Kaut JA, Hubbard SM, Longo DL, Simon RM, DeVita VT, Jaffe ES. The pathologic and clinical heterogeneity of lymphocyte-depleted Hodgkin's disease. J Clin Oncol 1986; 4: 284-294.

3. Cabanillas F, Fuller LM. The radiologic assessment of the lymphoma patient from the standpoint of the clinician. Radiol Clin North Am 1990; 28: 683-695.

4. Paryani SB, Hoppe RT, Cox RS, Colby TV, Rosenberg SA, Kaplan HS. Analysis of non-Hodgkin's lymphoma with nodular and favorable histologies. Cancer 1983; 52: 2300-2307.

5. Vokes EE, Uhmann JE, Golomb HM et al. Long-term survival of patients with localized diffuse histiocytic lymphoma. J Clin Oncol 1985; 3: 1309-1317.

6. Cmkovich MJ, Leopold K, Hoppe RT, Manch PM. Stage I to IIB Hodgkins disease: the combined experience at Stanford University and the Joint Center for Radiation Therapy. J Clin Oncol 1987; 5: 1041-1049.6. Strauss DJ. Strategies in the treatment of Hodgkin's disease. Senior Oncol 1986; 13: 26-34.

7. Coleman M. Chemotherapy for large-cell lymphoma: optimism and caution. Ann InternMed 1985; 103: 140142.

8. Ur Metser, Odelia Goor, Hedva Lerman, Elizabeth Naparstek, Einat Even-Sapir PET-CT of Extranodal Lymphoma. Am J Roentgenol 2004; 182: 1579-1586.

9. Armitage JO, Mauch PM, Harris NL, Bierman P. Lymphomas: extranodal sites. In: De Vita VT, Hellman S, Rosenberg SA, eds. Cancer: principles and practice of oncology, 6th ed. Philadelphia, PA: Lippincott Williams \& Wilkins, 2001: 2300-2316.

10. Moog F, Bangerter M, Diederichs CG et al. Extranodal malignant lymphoma: detection with FDG PET versus CT. Radiology 1998; 206: 475-481.

11. Hany TF, Steinert HC, Goerres GW, Buck A, von Schulthess GK. PET diagnostic accuracy: improvement with in-line PET-CT system-initial results. Radiology 2002; 225: 575-581.

12. Malloy PC, Fishman EK, Magid D. Lymphoma of bone, muscle, and skin: CT findings. Am J Roentgenol1992; 159: 805-809.

13. Rosenberg SA, Diamond HD, Taslowitz B, Craver LF. Lymphosarcoma: review of 1269 cases. Medicine 1961; 40: 31-84.

14. Buerger LF, Monteleone DN. Leukemic-lymphomatous infiltration of skeletal muscle: systematic study of 82 autopsy cases. Cancer 1966; 19: 1416-1422.

15. Freeman C, Berg JW, Cutler SJ. Occurrence and prognosis of extranodal lymphomas. Cancer 1972; 29: 252-260.

16. Ali Guermazi, Pauline Brice, Eric de Kerviler, Christophe Fermé, Christophe Hennequin, Véronique Meignin et al. Extranodal Hodgkin Disease: Spectrum of Disease RadioGraphics, Jan 2001; 21: 161-179.

17. Isohashi K, Tatsumi M, Higuchi I, Inoue A, Nakajo K et. al. 18F-FDG-PET in patients with malignant lymphoma having long-term follow-up: staging and restaging, and evaluation of treatment response and recurrence. Annals of Nuclear Medicine 2008; 22(9): 795-802. 
18. Allen-Auerbach M. de Vos S, Czernin J. The impact of fluorodeoxyglucose-positron emission tomography in primary staging and patient management in lymphoma patients. Radiologic Clinics of North America 2008; 46(2): 199-211.

19. Kirby AM, Mikhael NG. The role of FDG PET in the management of lymphoma: what is the evidence base? Nucl Med Commun 2007; 28(5):335-354.

20. Cheson BD, Pfister B, Juweid ME, Gascoyne RD, Specht L, Horning SJ et al. Revised Response Criteria for Malignant Lymphoma. J Clin Oncol 2007; 25: 579586.

21. Soudack M, Shalom RB, Israel O, Ben-Arie Y, Levy Z, Gaitini D. Utility of sonographically guided biopsy in metabolically suspected recurrent lymphoma. Journal of Ultrasound in Medicine 2008; 27(2): 225-31.

22. Kwee TC, Kwee RM, Nievelstein RA. Imaging in staging of malignant lymphoma: a systematic review. Blood. 2008; 111(2): 504-16.

23. Seam $P$, Juweid ME. Cheson BD. The role of FDG-PET scans in patients with lymphoma. Blood 2007; 110(10): 3507-16.

24. Podoloff DA, Advani RH, Allred C. Benson AB 3rd, Brown E, Burstein HJ et. al. NCCN task force report: positron emission tomography (PET)/computed tomography (CT) scanning in cancer. Journal of the National Comprehensive Cancer Network. 2007; 5 Suppl 1: S1-S22; quiz S23-2.

25. Juweid ME, Stroobants S, Hoekstra OS, Mottaghy FM, Dietlein M, Guermazi et. al. Use of positron emission tomography for response assessment of lymphoma: consensus of the Imaging Subcommittee of International Harmonization Project in Lymphoma.. Imaging Subcommittee of International Harmonization Project in Lymphoma. Journal of Clinical Oncology 2007; 25(5): 571-578.

26. Zinzani PL, Stefoni V, Tani M, Fanti S, Musuraca G, Castellucci et. al. Role of [18F] fluorodeoxyglucose positron emission tomography scan in the follow-up of lymphoma. Journal of Clinical Oncology 2009; 27(11): 1781-1787.

27. Levine E, Lee KR, Neff JR et al: Comparison of computed tomography and other imaging modalities in the evaluation of musculoskeletal tumors. Radiology 1979; 131: 431-437.

28. Grem JL, Neville AJ, Smith SC, Gould HR. Love RR, Trump DL. Massive skeletal muscle invasion by lymphoma. Arch Intern Med 1985; 145: 1818-1820.

29. Scally J, Garrett A. Primary extranodal lymphoma in muscle. Br J Radiol 1989; 62: 81.

30. Schwalke MA, RodiljV, Vezeridis MP. Primary lymphoma arising in skeletal muscle. Eur J Surg Oncol 1990; 16: 70-73.
31. Pileri S, Serra L, Bazzocchi F, Martinelli C, Govoni E, Seven B. Linfoma maligno clinicamente pnimitivo del musculo scheletico (presentazione di un caso e revisione della letteratura). Pathologica 1979; 71 : 485-494.

32. Kandel RA, Bdard YC, Pritzker KPH, Luk SC. Lymphoma presenting as an intramuscular small cell malignant tumor. Cancer 1984; 53: 1586-1589.

33. Hong-Jen Chiou, Yi-Hong Chou, See-Ying Chiou, et al. High-Resolution Ultrasonography of Primary Peripheral Soft Tissue Lymphoma. J Ultrasound Med 2005; 24: 77-86 • 0278-4297.

34. Suresh S, Saifuddin A, O'Donnell P. Lymphoma presenting as a musculoskeletal soft tissue mass: MRI findings in 24 cases. Eur Radio 2008; 18: 2628-2634.

35. Lale Kostakoglu, Leonard JP, Ichiei Kuji et. al. Comparison of fluorine-18 fluorodeoxyglucose positron emission tomography and Ga-67 scintigraphy in evaluation of lymphoma. Cancer 2002; 94: 879-88.

36. Spaepen K, Stroobants S, Dupont P et al: Prognostic value of positron emission tomography (PET) with fluorine-18 fluorodeoxyglucose ([18F]FDG) after first-line chemotherapy in non-Hodgkin's lymphoma: Is [18F] FDG-PET a valid alternative to conventional diagnostic methods? J Clin Oncol 2001; 19: 414-419.

37. Spaepen K, Stroobants $S$, Dupont $P$ et al: Early restaging positron emission tomography with $18 \mathrm{~F}$ fluorodeoxyglucose predicts outcome in patients with aggressive non-Hodgkin's lymphoma. Ann Oncol 2002; 13: 1356-1363.

38. Haioun C, Itti E, Rahmouni A, et al: [18F]fluoro-2-deoxyD-glucose positron emission tomography (FDG-PET) in aggressive lymphoma: An early prognostic tool for predicting patient outcome. Blood 2005; 106: 13761381.

39. Gallamini A, Rigacci L, Merli F et al: Predictive value of positron emission tomography performed after two courses of standard therapy on treatment outcome in advanced stage Hodgkin's disease. Haematologica 2006; 91: 475-481.

40. Kostakoglu L, Coleman M, Leonard JP et al: PET predicts prognosis after 1 cycle of chemotherapy in aggressive lymphoma and Hodgkin's disease. J Nucl Med 2002; 43: 1018-1027.

41. Zinzani PL, Tani M, Fanti S et al: Early positron emission tomography (PET) restaging: A predictive final response in Hodgkin's disease patients. Ann Oncol 2006; 17: 1296-1300.

42. Samuel LM, White J, Lessells AM, Roddie H, Matheson LM. Primary non-Hodgkins lymphoma of muscle. Clin Oncol (R Coll Radiol) 1999.

43. Paes FM, Kalkanis DG, Sideras PA, Serafini AN. RSNA 2010 FDG PET/CT of Extranodal Involvement in Non-Hodgkin Lymphoma and Hodgkin Disease. 